\title{
Differential Equations in the Spectral Parameter, Darboux Transformations and a Hierarchy of Master Symmetries for KdV
}

\author{
Jorge P. Zubelli ${ }^{1, \star}$ and Franco Magri ${ }^{2, \star \star}$ \\ Department of Mathematics, University of California, Berkeley, CA 94720, USA \\ 2 Mathematical Sciences Research Institute, University of California, Berkeley,CA 94720, USA \\ Received November 29, 1990; in revised form April 30, 1991
}

\begin{abstract}
We study a certain family of Schrödinger operators whose eigenfunctions $\varphi(x, \lambda)$ satisfy a differential equation in the spectral parameter $\lambda$ of the form $B\left(\lambda, \partial_{\lambda}\right) \varphi=\Theta(x) \varphi$. We show that the flows of a hierarchy of master symmetries for $\mathrm{KdV}$ are tangent to the manifolds that compose the strata of this class of bispectral potentials. This extends and complements a result of Duistermaat and Grünbaum concerning a similar property for the Adler and Moser potentials and the flows of the $\mathrm{KdV}$ hierarchy.
\end{abstract}

\section{Introduction}

The theory of solitons is still a source of surprises and unexpected connections. The purpose of this article is to report another one of these connections. More specifically, the link between a hierarchy of nonlinear evolution equations closely related to the Korteweg-de Vries (KdV) equation and the bispectral problem, which was introduced in [8]. This problem, for the Schrödinger operator $L=-\partial_{x}^{2}+u$, can be formulated as follows: When do the solutions $\varphi(x, \lambda)$ of

$$
L \varphi=\lambda \varphi
$$

also satisfy a differential equation in the spectral parameter $\lambda$ of the form

$$
B\left(\lambda, \partial_{\lambda}\right) \varphi=\Theta(x) \varphi,
$$

where $B\left(\lambda, \partial_{\lambda}\right)$ is a differential operator of positive order and $\Theta(x)$ is independent of $\lambda$ ? The solution to this problem, under very mild assumptions on $u(x)$, turns out to be related to the theory of the $\mathrm{KdV}$ equation

$$
u_{t}=-u_{x x x}+6 u_{x} u
$$

* Present address: Department of Mathematics, University of California, Santa Cruz, CA 95064, USA

$\star \star$ Present address: Departimento di Matematica, Università di Milano, I-20133 Milano, Italy 
in a very peculiar form. As shown in [8], the bispectral potentials that are bounded $^{1}$ at $x=\infty$ can be divided into two classes according to the dimension of the space of common solutions of Eqs. (1) and (2). This space can have dimension 1 or 2 . If there exists a pair $(B, \Theta)$ such that this space is two-dimensional, we shall say that the potential is a rank two bispectral potential. The remaining bispectral potentials are called rank one. These last ones had been previously studied by Adler, Airault, McKean, and Moser [1,2] in a different context. They have the remarkable property of remaining rational under the flows of the $\mathrm{KdV}$ hierarchy, from which Eq. (3) is the first nontrivial example. Although the rank two bispectral potentials were completely characterized via rational Darboux transformations in [8], they have always been much more mysterious than its complement. One reason is that the rank two potentials did not appear explicitly in any previous work. Another one is the fact that, in general, they do not remain rational under the flows of the $\mathrm{KdV}$ hierarchy. The main contribution of the present work is to establish the invariance of the class of rank two bispectral potentials, and of certain manifolds contained therein, by the flows of a hierarchy of nonlinear evolution equations intimately related to $\mathrm{KdV}$. This hierarchy has a number of interesting properties, some of which we shall describe in Sect. 2. It is composed of master symmetries [10] for the $\mathrm{KdV}$ hierarchy and it also appeared in the work of Levi [20].

In order to describe our results in more detail we need to introduce another key element in the picture. This element is the concept of rational Darboux transformations. By a Darboux transformation we mean the process of constructing from $L=-\partial_{x}^{2}+u$ and $\phi \in \operatorname{ker} L$ another operator $\widetilde{L}=-\partial_{x}^{2}+\tilde{u}$ such that

$$
\tilde{u}=u-2 \partial_{x}^{2} \log \phi \text {. }
$$

This corresponds to factoring the operator $L$ into $A^{*} A$, where $A$ and $A^{*}$ are first order differential operators, and setting $\tilde{L}=A A^{*}$. In Sect. 3 we present a more detailed description of this important transformation that appeared in a number of different contexts, $[1,5-7,21,25]$ to cite just a few. If the potentials $u$ and $\tilde{u}$ are both rational functions of $x$, we call the corresponding Darboux transformation a rational one. We remark that given a rational function $u$, it is extremely unlikely that $\tilde{u}$ will also be rational. Notwithstanding, this phenomenon appears throughout the study of the bispectral problem. In fact, the potentials that stay rational by the KdV flows can be characterized as the result of successive applications of rational Darboux transformations to potentials of the form $v(v-1) / x^{2}$, with $v \in \mathbf{Z}_{>0}$. The rank two classes can be obtained by the union of the set of potentials of the form $c / x^{2}$ with those constructed by rational Darboux transformations applied to $\left(l^{2}-1 / 4\right) / x^{2}$, with $l \in \mathbf{Z}_{>0}$. Since each time we apply Darboux a new parameter is introduced we can look at the potentials resulting of $n$ Darboux transformations as an $n$-dimensional complex manifold. The following questions arise naturally:

1. Is there some hierarchy of nonlinear evolution equations whose flows preserve the rationality of the potentials in the rank two class?

2. If so, are the potentials in the orbit of such flows still bispectral?

3. Is this hierarchy related to some completely integrable system and can it be described in terms of some recursion operator?

1 This restriction of boundedness at $\infty$ is actually immaterial here. If it is removed, the only potentials that have to be added to the list are the ones of the form $u(x)=a x+b$ [8] 
Our answers to these questions are in terms of a hierarchy evolution equations defined via the recursion operator

$$
\mathbf{N}_{u}=-\partial_{x}^{2}+4 u+2 u_{x} \partial_{x}^{-1},
$$

which gives rise to the higher order KdV flows

$$
X_{j}(u)=\mathbf{N}_{u}^{j} u_{x} .
$$

We start with

$$
\tau_{0}(u)=\frac{1}{2} x u_{x}+u
$$

and define

$$
\tau_{j}(u)=\mathbf{N}_{u}^{j} \tau_{0}(u) .
$$

The hierarchy $\left\{\tau_{j}\right\}_{j=0}^{\infty}$ answers the first and the second questions affirmatively, see Sect. 4. The answer to the third question, which is also positive, is developed in Sect. 2.

We present a few examples in order to illustrate the next sections and the bispectral property. The most simple examples of bispectral potentials are those of the form

$$
u(x)=\frac{c}{x^{2}},
$$

with $c \in \mathbf{C}$. It is easy to see that if we take $\Phi(z)$ a solution of

$$
-\Phi^{\prime \prime}+\frac{c}{z^{2}} \Phi=\Phi,
$$

and define

$$
\varphi(x, \lambda)=\Phi\left(x \lambda^{1 / 2}\right)
$$

then

$$
L \varphi=\lambda \varphi
$$

and

$$
\left[-\left(2 \lambda^{1 / 2} \partial_{\lambda}\right)^{2}+\frac{c}{\lambda}\right] \varphi=x^{2} \varphi .
$$

Since the function $\Phi$ is an arbitrary solution of (10), the space of common solutions of (12) and (13) has dimension 2. Hence, $c / x^{2}$ belongs to the rank two family of bispectral potentials. It is easy to check that $c / x^{2}$ is a stationary solution of $\partial_{t} u$ $=\tau_{0}(u)$. The next example is given by

$$
u_{1}=\frac{l^{2}-1 / 4}{x^{2}}-2 \partial_{x}^{2} \log \left(x^{-l+1 / 2}+t_{0} x^{l+1 / 2}\right) .
$$

It was shown in [8] that the potential $u_{1}$ above is bispectral. It is obtained by applying one Darboux transformation to $u_{0}=\left(l^{2}-1 / 4\right) / x^{2}$, as will be shown in Sect. 3. A straightforward computation shows that

$$
\tau_{0}\left(u_{1}\right)=t_{0} l \frac{\partial u_{1}}{\partial t_{0}}
$$


and

$$
\tau_{1}\left(u_{1}\right)=0
$$

Hence, $\tau_{j}\left(u_{1}\right)=0$ for $j \geqq 1$. In other words, the vector fields $\tau_{j}, j \in \mathbf{Z}_{>0}$, are all tangent to the one-dimensional manifold of potentials obtained by applying Darboux to $u_{0}$. Theorem 8 extends this result to an arbitrary number of Darboux transformations.

The plan for this work is the following:

In Sect. 2 we develop the theory of the hierarchy of vector fields $\left\{\tau_{j}\right\}_{j=0}^{\infty}$. We also describe the corresponding hierarchy associated to the $\mathrm{mKdV}$ equation. In Sect. 3 we study the potentials $u_{n}$ obtained by $n$ Darboux transformations to $u_{0}=\left(l^{2}-1 / 4\right) / x^{2}$. Such potentials depend on $n$ parameters and form certain $n$-dimensional manifolds, which we denote by $M_{n}$. In Sect. 4 we prove the main result of this paper, namely, that the vector fields $\tau_{j}, j=0,1, \ldots$, are tangent to the manifold $M_{n}$.

\section{A Hierarchy of Master Symmetries Associated to KdV}

\subsection{Preliminaries}

In this section we collect a few properties of hierarchies of nonlinear evolution equations connected with the $\mathrm{KdV}$ equation

$$
u_{t}=-u_{x x x}+6 u_{x} u \stackrel{\text { def }}{=} X_{1}(u) \text {. }
$$

Our approach is geometric and based on recursion operators [22-24, 29]. We look at the right-hand side of this equation as a vector field defined on a vector space $\mathscr{U}$. For our purposes it is convenient to take $\mathscr{U}$ to be a space of functions $u(x)$ satisfying the following two properties:

1. The function $u(x)$ is holomorphic in an open sector $S$ containing an unbounded subinterval of the negative real axis. (The sector $S$ depends on the function $u$.)

2. The asymptotic behavior of $u(x)$, as $x \rightarrow \infty$, is ${ }^{2}$

$$
u(x)=\mathcal{O}\left(\frac{1}{x^{2}}\right), \quad x \in S .
$$

The usual space where the formalism of recursion operators is employed is a space of rapidly decaying $C^{\infty}$ functions on the line. Our choice of spaces is motivated by the fact that we will be working with rational functions of $x$ and with functions that are rational in $\log x$ and $x^{1 / 2}$. Henceforth, we choose a branch of the logarithm with a cut coincident to the positive real axis.

We proceed with two important remarks. The first one is that we can write

$$
X_{1}(u)=\mathbf{N}_{u} X_{0}(u),
$$

where $X_{0}(u) \stackrel{\text { def }}{=} u_{x}$, and $\mathbf{N}_{u}$ is a linear map, depending on $u \in \mathscr{U}$, defined by

$$
\mathbf{N}_{u} \psi=-\partial_{x}^{2} \psi+4 u \psi+2 u_{x} \partial_{x}^{-1} \psi
$$

${ }_{2}$ Throughout this paper, as usual, $f(x)=\mathcal{O}(g(x)), x \rightarrow a$, means that $f(x) / g(x)$ is bounded near to $a$ for $x \in S$ 
for $\psi \in \mathscr{U}$. Here, by $\partial_{x}^{-1} \psi$ we mean

$$
\partial_{x}^{-1} \psi=\int_{\infty}^{x} \psi(s) d s,
$$

where the path of integration connecting $\infty$ to $x$ lies in $S$. Notice that, since $u \in \mathscr{U}$, we have that $u_{x}=\mathcal{O}\left(1 / x^{3}\right)$ and $\partial_{x}^{-1} u=\mathcal{O}(1 / x)$ for $x$ in any subsector whose rays are in the interior of $S$. The proof of this is a straightforward adaptation of Theorems 8.7 and 8.8, Chap. 3, of [31]. We shall now state some properties of $\mathbf{N}_{u}$ considered as a tensor field of type $(1,1)$ on $\mathscr{U}$. For a pair of constant vector fields $\varphi$ and $\psi$ in $\mathscr{U}$ and an arbitrary vector field $X(u)$ we define the directional derivatives

$$
\left.X_{u}^{\prime}(\varphi) \stackrel{\text { def }}{=} \frac{d}{d \varepsilon} X(u+\varepsilon \varphi)\right|_{\varepsilon=0}
$$

and

$$
\left.\mathbf{N}_{u}^{\prime}(\varphi ; \psi) \stackrel{\text { def }}{=} \frac{d}{d \varepsilon} \mathbf{N}_{u+\varepsilon \varphi} \varphi\right|_{\varepsilon=0} .
$$

For $\mathbf{N}_{u}$ we have explicitly

$$
\mathbf{N}_{u}^{\prime}(\varphi ; \psi)=4 \varphi \psi+2 \psi_{x} \partial_{x}^{-1} \varphi .
$$

The Nijenhuis torsion of $\mathbf{N}_{u}$ is the vector valued 2-form given by

$$
\mathbf{T}_{u}(\varphi, \psi)=\mathbf{N}_{u}^{\prime}\left(\psi, \mathbf{N}_{u} \varphi\right)-\mathbf{N}_{u}^{\prime}\left(\varphi, \mathbf{N}_{u} \psi\right)+\mathbf{N}_{u} \mathbf{N}_{u}^{\prime}(\varphi, \psi)-\mathbf{N}_{u} \mathbf{N}_{u}^{\prime}(\psi, \varphi) .
$$

Notice that $\mathbf{T}_{u}$ is skew-symmetric in $(\varphi, \psi)$. In fact, Eq. (17) is a particular case of a more general definition of the Nijenhuis torsion [9] of a tensor of type $(1,1)$ on a manifold. If $X$ and $Y$ are vector fields on this manifold and $G$ is a tensor field of type $(1,1)$, the torsion of $G$ is a vector valued 2 -form defined by

$$
\mathbf{T}_{u}(X, Y) \stackrel{\text { def }}{=}[G X, G Y]-G[X, G Y]-G[G X, Y]+G^{2}[X, Y],
$$

where the bracket $[\cdot, \cdot]$ denotes the vector field commutator $[3,16,18]$

$$
[X, Y](u)=Y_{u}^{\prime} X(u)-X_{u}^{\prime} Y(u) .
$$

If the manifold is also a vector space we can consider the constant vector fields given by $X(u)=\varphi$ and $Y(u)=\psi$, where $\varphi$ and $\psi$ are fixed elements of the underlying vector space. By using Eq. (19) it is easy to obtain Eq. (17) from (18). It is also easy to verify that for $\mathbf{N}_{u}$ given by (15), the torsion

$$
\mathbf{T}_{u}(\varphi, \psi)=0 .
$$

Since the torsion is a tensorial quantity it also vanishes for arbitrary vector fields, provided that we use now Eq. (18) to compute it. Recall that the Lie derivative of the $(1,1)$ tensor $\mathbf{N}_{u}$ along the vector field $X(u)$ is a tensor field of the same type defined by

$$
\mathscr{L}_{X}\left(\mathbf{N}_{u}\right) \varphi=\mathbf{N}_{u}^{\prime}(\varphi ; X)+\mathbf{N}_{u} X_{u}^{\prime} \varphi-X_{u}^{\prime} \mathbf{N}_{u} \varphi .
$$

The second important remark is that for $\mathbf{N}_{u}$ given by (15) and $X_{0}(u)=u_{x}$, we have that

$$
\mathscr{L}_{X_{0}}\left(\mathbf{N}_{u}\right)=0 .
$$


So, we can say that $X_{0}$ is an infinitesimal symmetry of $\mathbf{N}_{u}$. fields

The consequence of the two remarks above is the following: The iterated vector

$$
X_{j} \stackrel{\text { def }}{=} \mathbf{N}_{u}^{j} X_{0}
$$

satisfy $\left[X_{j}, X_{l}\right]=0$.

The vector fields $X_{j}$ generate the higher order $\operatorname{KdV}$ flows $[29,23,24]$

$$
\partial_{t_{j}} u=X_{j}(u) .
$$

These flows can also be obtained via the Lax pair formalism [19] and by fractional powers of differential operators. See $[12,13,30]$ and references therein for a more comprehensive list of references on such approaches.

\subsection{Master Symmetries}

We shall now construct, from our point of view, a hierarchy of master symmetries for the KdV equation. The concept of master symmetries was introduced by Fuchssteiner and was applied to a number of important examples such as the Benjamin-Ono equation and the K.P. hierarchy [10, 11, 27, 28].

Consider the vector field

$$
\tau_{0}(u)=\frac{1}{2} x u_{x}+u .
$$

If we compute the Lie derivative of $\mathbf{N}_{u}$, with respect to $\tau_{0}$, we find

$$
\mathscr{L}_{\tau_{0}}\left(\mathbf{N}_{u}\right)=\mathbf{N}_{u} .
$$

Leibniz rule gives

$$
\mathscr{L}_{\tau_{0}}\left(\mathbf{N}_{u}^{j}\right)=j \mathbf{N}_{u}^{j} .
$$

Due to Eq. (20), by analogy with the definition of a conformal symmetry of a Riemannian metric, it seems natural to call $\tau_{0}$ a conformal symmetry of $\mathbf{N}_{u}$. Let's now consider the hierarchy $\left\{\tau_{j}\right\}_{j=0}^{\infty}$ obtained by defining

$$
\tau_{j}=\mathbf{N}_{u}^{j} \tau_{0} .
$$

The vector fields $\tau_{j}$, for $j \geqq 0$, do not commute with one another, since $\tau_{0}$ is not a symmetry of $\mathbf{N}_{u}$. However, they do verify the following remarkable commutation relation

$$
\left[\tau_{j}, \tau_{l}\right]=(l-j) \tau_{j+l},
$$

as will be shown below. The reader familiar with Virasoro algebras will recognize Eq. (22) as defining a Virasoro algebra of zero central charge.

To prove Eq. (22) we start by remarking that such objects are well defined in the space $\mathscr{U}$, since $\tau_{0}(\mathscr{U}) \subset \mathscr{U}$ and $\mathbf{N}_{u}(\mathscr{U}) \subset \mathscr{U}$. Secondly, we prove that the vanishing of the torsion of $\mathbf{N}_{u}$ yields

$$
\mathscr{L}_{\mathbf{N}_{u} X}\left(\mathbf{N}_{u}\right)=\mathbf{N}_{u} \mathscr{L}_{X}\left(\mathbf{N}_{u}\right),
$$

and hence

$$
\mathscr{L}_{\mathbf{N}_{u}^{j} X}\left(\mathbf{N}_{u}\right)=\mathbf{N}_{u}^{j} \mathscr{L}_{X}\left(\mathbf{N}_{u}\right) .
$$


Indeed, from the vanishing of the torsion and by Leibniz rule

$$
\begin{aligned}
0 & =\left[\mathbf{N}_{u} X, \mathbf{N}_{u} Y\right]-\mathbf{N}_{u}\left[\mathbf{N}_{u} \mathbf{X}, Y\right]-\mathbf{N}_{u}\left[X, \mathbf{N}_{u} Y\right]+\mathbf{N}_{u}^{2}[X, Y] \\
& =\mathscr{L}_{\mathbf{N}_{u} X}\left(\mathbf{N}_{u} Y\right)-\mathbf{N}_{u} \mathscr{L}_{\mathbf{N}_{u} X}(Y)-\mathbf{N}_{u} \mathscr{L}_{X}\left(\mathbf{N}_{u} Y\right)+\mathbf{N}_{u}^{2} \mathscr{L}_{X}(Y) \\
& =\left(\mathscr{L}_{\mathbf{N}_{u} X}\left(\mathbf{N}_{u}\right)-\mathbf{N}_{u} \mathscr{L}_{X}\left(\mathbf{N}_{u}\right)\right) Y .
\end{aligned}
$$

Now, we compute

$$
\begin{aligned}
{\left[\tau_{j}, \tau_{l}\right] } & =\mathscr{L}_{\tau_{j}}\left(\mathbf{N}_{u}^{l} \tau_{0}\right) \\
& =\mathscr{L}_{\tau_{j}}\left(\mathbf{N}_{u}^{l}\right) \tau_{0}+\mathbf{N}_{u}^{l} \mathscr{L}_{\tau_{j}}\left(\tau_{0}\right) \\
& =\mathscr{L}_{\mathbf{N}_{u}^{j} \tau_{0}}\left(\mathbf{N}_{u}^{l}\right) \tau_{0}-\mathbf{N}_{u}^{l} \mathscr{L}_{\tau_{0}}\left(\mathbf{N}_{u}^{j} \tau_{0}\right) \\
& =\mathscr{L}_{\mathbf{N}_{u}^{j} \tau_{0}}\left(\mathbf{N}_{u}^{l}\right) \tau_{0}-\mathbf{N}_{u}^{l} \mathscr{L}_{\tau_{0}}\left(\mathbf{N}_{u}^{j}\right) \tau_{0}-\mathbf{N}_{u}^{l+j} \mathscr{L}_{\tau_{0}} \tau_{0} \\
& =\mathscr{L}_{\mathbf{N}_{u} \tau_{0}}\left(\mathbf{N}_{u}^{l}\right) \tau_{0}-\mathbf{N}_{u}^{l} \mathscr{L}_{\tau_{0}}\left(\mathbf{N}_{u}^{j}\right) \tau_{0} .
\end{aligned}
$$

From Eqs. (24) and (21) we get

$$
\begin{aligned}
{\left[\tau_{j}, \tau_{l}\right] } & =\mathbf{N}_{u}^{j} \mathscr{L}_{\tau_{0}}\left(\mathbf{N}_{u}^{l}\right) \tau_{0}-\mathbf{N}_{u}^{l} \mathscr{L}_{\tau_{0}}\left(\mathbf{N}_{u}^{j}\right) \tau_{0} \\
& =\left(\mathbf{N}_{u}^{j} l \mathbf{N}_{u}^{l}-\mathbf{N}_{u}^{l} j \mathbf{N}_{u}^{j}\right) \tau_{0} \\
& =(l-j) \tau_{j+l} .
\end{aligned}
$$

The first two nonlinear master symmetry fields constructed above are given by

$$
\begin{gathered}
\tau_{1}(u)=-\frac{x}{2}\left(u_{x x x}-6 u u_{x}\right)-2 u_{x x}+u_{x} \partial_{x}^{-1} u+4 u^{2}, \\
\tau_{2}(u)=\frac{x}{2}\left(u_{5 x}-10 u u_{3 x}-18 u_{x} u_{x x}+24 u^{2} u_{x}\right)+3 u_{4 x}-u_{3 x} \partial_{x}^{-1} u \\
-24 u u_{x x}-15 u_{x}^{2}+u_{x}\left(4 u \partial_{x}^{-1} u+2 \partial_{x}^{-1} \tau_{1}(u)\right)+16 u^{3} .
\end{gathered}
$$

In order to fully appreciate the power of the remark that the torsion of $\mathbf{N}_{u}$ vanishes, the reader should try to prove Eq. (22) for $0 \leqq l, j \leqq 2$ directly!

The master symmetry vector fields defined above are deeply connected to the $\mathrm{KdV}$ flows. To show this we first compute the commutator of $X_{0}$ and $\tau_{0}$, which gives

$$
\left[X_{0}, \tau_{0}\right]=-\frac{1}{2} X_{0} .
$$

Thus, by repeating the argument given above for $\left[\tau_{j}, \tau_{l}\right]$ we find that

$$
\begin{aligned}
{\left[X_{j}, \tau_{l}\right] } & =\mathscr{L}_{\mathbf{N}_{u}^{j} X_{0}}\left(\mathbf{N}_{u}^{l} \tau_{0}\right) \\
& =\mathscr{L}_{\mathbf{N}_{u}^{j} X_{0}}\left(\mathbf{N}_{u}^{l}\right) \tau_{0}+\mathbf{N}_{u}^{l} \mathscr{L}_{\mathbf{N}_{u}^{j} X_{0}}\left(\tau_{0}\right) \\
& =\mathbf{N}_{u}^{j} \mathscr{L}_{X_{0}}\left(\mathbf{N}_{u}^{l}\right) \tau_{0}-\mathbf{N}_{u}^{l} \mathscr{L}_{\tau_{0}}\left(\mathbf{N}_{u}^{j}\right) X_{0}-\mathbf{N}_{u}^{l+j} \mathscr{L}_{\tau_{0}}\left(X_{0}\right) \\
& =-\mathbf{N}_{u}^{l} \mathscr{L}_{\tau_{0}}\left(\mathbf{N}_{u}^{j}\right) X_{0}-\mathbf{N}_{u}^{l+j} \mathscr{L}_{\tau_{0}}\left(X_{0}\right) \\
& =-j \mathbf{N}_{u}^{l+j} X_{0}-\frac{1}{2} \mathbf{N}_{u}^{l+j} X_{0} \\
& =\left(-j-\frac{1}{2}\right) X_{l+j} .
\end{aligned}
$$

In going from Eq. (29) to (30) we used Eqs. (21) and (25). The conclusion from Eq. (31) is that the master symmetry fields can be used to generate, via commutators, the fields in the KdV hierarchy. We summarize the results thus far in the following: ${ }^{3}$

3 Proposition 1 is part of a more general theory of master symmetries. See $[4,26]$ and references therein 
Proposition 1. The hierarchy $\left\{\tau_{j}\right\}_{j=0}^{\infty}$ satisfies

$$
\left[\tau_{j}, \tau_{l}\right]=(l-j) \tau_{j+l}
$$

and

$$
\left[X_{j}, \tau_{l}\right]=-\left(j+\frac{1}{2}\right) X_{l+j}
$$

\subsection{Transformation Properties}

We shall now consider the relation of the KdV hierarchy with the mKdV one. It is well known that the Miura map

$$
u=F(v) \stackrel{\text { def }}{=} v^{2}+v_{x}
$$

is a transformation that maps solutions of the $\mathrm{mKdV}$ equation

$$
v_{t}=-v_{x x x}+6 v^{2} v_{x} \stackrel{\text { def }}{=} Y_{1}(v)
$$

into solutions of the KdV equation (14). We shall look at (33) as a vector field in the space $\mathscr{V}$ of holomorphic functions defined in a sector near $\infty$, around the negative real axis, and with asymptotic behavior of the form $\mathcal{O}(1 / x)$. We remark that the Miura map $v \mapsto F(v)$ is a point transformation from $\mathscr{V}$ into $\mathscr{U}$. Its directional derivative is given by

$$
F_{v}^{\prime} \dot{v}=2 v \dot{v}+\dot{v}_{x}
$$

The corresponding transformation law for vector fields is

$$
X(F(v))=F_{v}^{\prime} . Y(v) .
$$

In particular, the vector fields $X_{1}$ and $Y_{1}$ defined above are $F$-related. Next, we exhibit a torsionless tensor field of type $(1,1)$ on $\mathscr{V}$, which is $F$-related to the tensor $\mathbf{N}_{u}$ defined in Eq. (15). It is given by the following linear map:

$$
\mathbf{M}_{v}: \psi \mapsto \mathbf{M}_{v} \psi \stackrel{\text { def }}{=} \partial_{x}\left(-\partial_{x} \psi+4 v \partial_{x}^{-1}(v \psi)\right)
$$

Remark that if $v$ and $\psi$ are in $\mathscr{V}$, so is $\mathbf{M}_{v} \psi$. One can easily check that, for $u=F(v)$,

$$
\mathbf{N}_{u} \cdot F_{v}^{\prime}=F_{v}^{\prime} \cdot \mathbf{M}_{v} .
$$

Consequently, the torsion of $\mathbf{M}_{v}$ and $\mathbf{N}_{u}$ are $F$-related. A straightforward computation gives that the torsion of $\mathbf{M}_{v}$ vanishes. We also remark that if we define the vector fields $Y_{0}$ and $\sigma_{0}$ by

$$
Y_{0}(v) \stackrel{\text { def }}{=} v_{x}
$$

and

$$
\sigma_{0}(v)=\frac{1}{2}(x v)_{x},
$$

then $Y_{0}$ and $\sigma_{0}$ are $F$-related to $X_{0}$ and $\tau_{0}$, respectively. Once again, $Y_{0}$ is a symmetry of $\mathbf{M}_{v}$ and $\sigma_{0}$ is a conformal symmetry of $\mathbf{M}_{v}$. The $\mathrm{mKdV}$ hierarchy is obtained by

$$
Y_{j} \stackrel{\text { def }}{=} M_{v}^{j} Y_{0}
$$


and the associated master symmetry hierarchy by

$$
\sigma_{j} \stackrel{\text { def }}{=} \mathbf{M}_{v}^{j} \sigma_{0} \text {. }
$$

Obviously, these hierarchies are also $F$-related to the corresponding ones in the "KdV domain" and satisfy equations similar to (22) and (25). We close this section by calling attention to the fact that $\mathbf{M}_{v} \psi=\mathbf{M}_{-v} \psi$. This will play an important role in the final steps of the proof of Theorem 8.

\section{Darboux Transformations and Families of Bispectral Potentials}

\subsection{Outline of this Section and Preliminary Remarks}

The first goal of this section is to describe the potentials $u_{n}$ obtained by $n$ successive applications of the Darboux transformations to

$$
L_{0}=-\partial_{x}^{2}+u_{0}
$$

where $u_{0}=\left(l^{2}-1 / 4\right) / x^{2}$ and $l \in \mathbf{Z}_{>0}$. As we shall see, each Darboux transformation introduces a new complex parameter in the resulting potential. By introducing such parameters in a suitable way we shall construct an $n$-dimensional complex manifold $M_{n}$, given by ${ }^{4}$

$$
M_{n} \stackrel{\text { def }}{=}\left\{u_{n}\left(\cdot ; t_{0}, \ldots, t_{n-1}\right) \mid\left(t_{0}, \ldots, t_{n-1}\right) \in \mathbf{C}^{n}\right\} .
$$

If $n \leqq l$, then $u_{n}$ is a rational function of $x$. It is a consequence of [8] that there exists a differential operator $B\left(k, \partial_{k}\right)$ and a function $\Theta(x)$ such that the space of common solutions of

$$
\left(-\partial_{x}^{2}+u_{n}\right) \varphi=k^{2} \varphi
$$

and

$$
B\left(k, \partial_{k}\right) \varphi=\Theta(x) \varphi
$$

has dimension 2. Furthermore, any rank two bispectral potential, modulo translation and addition of a constant, belongs to the union of $\bigcup_{l>0} \bigcup_{0 \leqq n \leqq l} M_{n, l}$ with the set of potentials of the form $c / x^{2}$ and $a x+b$, where $a, b$, and $c$ are arbitrary constants [8].

This section was influenced by the exposition in [32], which unfortunately was not made available to a larger audience. This brings in the second goal of this section, which is to extend the construction of the manifolds $M_{n, l}$ for $n>l$. This was not done in [32]. For such values of $n, M_{n, l}$ has elements that are not rational anymore. However, we shall prove the peculiar fact that the potentials in this case are rational functions in $x^{1 / 2}$ and $\log x$. Since, as shown in [8], the bispectral potentials are always rational functions of $x$, for $n>l$ the manifolds $M_{n, l}$ are not composed of bispectral potentials anymore. The interesting fact is that the tangency result of Sect. 4 remains in this case.

\footnotetext{
${ }^{4}$ Strictly speaking $u_{n}$ and $M_{n}$ are also dependent on $l$, and we should write $u_{n, l}$ and $M_{n, l}$. However, we will do this only when it is unavoidable
} 
The plan for this section is the following:

In Sect. 3.2 we give the general description of applying $n$ Darboux transformations to $u_{0}=\left(l^{2}-1 / 4\right) / x^{2}$. In this paragraph this is done without specifying the choices of the element in $\operatorname{ker} L_{n-1}$ at the $n^{\text {th }}$ step. This construction is completed in Sect. 3.4 with the help of the concept of dominant and recessive solutions. In Sect. 3.3 we collect a few general facts about the intertwining operator $U_{n}$ such that $L_{n} U_{n}=U_{n} L_{0}$. In Sect. 3.5 we prove some results about the functions $v_{n}$, which are related to $u_{n}$ via the Miura map.

\subsection{The Construction}

We shall first establish some terminology. In what follows, $A$ will denote the firstorder differential operator

$$
A=\partial_{x}-v
$$

and $A^{*}$ its formal adjoint

$$
A^{*}=-\partial_{x}-v
$$

If we take

$$
v=\chi^{\prime} / \chi
$$

where $\chi$ is a solution of

$$
L \chi=\left(-\partial_{x}^{2}+u\right) \chi=0
$$

then

$$
L=A^{*} A \text {. }
$$

We remark that the relation between $v$ and $u$ here is given by the Miura map $u=v^{2}+v_{x}$. We start from $L_{0}$ as in Eq. (34), and $\chi_{0}$ a nonzero element in $\operatorname{ker} L_{0}$. This gives a factorization

$$
L_{0}=A_{0}^{*} A_{0}
$$

with $A_{0}, A_{0}^{*}$, and $v$ as in Eqs. (37), (38), and (39). Take

$$
L_{1} \stackrel{\text { def }}{=} A_{0} A_{0}^{*} \text {. }
$$

A simple computation shows that $L_{1}=-\partial_{x}^{2}+u_{1}$, with

$$
u_{1}=u_{0}-2 \partial_{x}^{2} \log \chi_{0} .
$$

Suppose we have found pairs $\left(\chi_{0}, L_{0}\right), \ldots,\left(\chi_{n-2}, L_{n-2}\right)$ such that

$$
L_{j}=A_{j}^{*} A_{j} \text {, for } 0 \leqq j \leqq n-2,
$$

and

$$
L_{j}=A_{j-1} A_{j-1}^{*}, \quad \text { for } \quad 0<j \leqq n-1 .
$$

We take $\chi_{n-1} \in \operatorname{ker} L_{n-1} \backslash\{0\}$, set

$$
\begin{aligned}
& A_{n-1} \stackrel{\text { def }}{=} \partial_{x}-v_{n-1}, \\
& v_{n-1} \stackrel{\text { def }}{=} \chi_{n-1}^{\prime} / \chi_{n-1},
\end{aligned}
$$


and

$$
L_{n} \stackrel{\text { def }}{=} A_{n-1} A_{n-1}^{*} \text {. }
$$

Notice that our choice of $\chi_{n-1} \in \operatorname{ker} L_{n-1}$ gives $L_{n-1}=A_{n-1}^{*} A_{n-1}$. We can illustrate this process via the following diagram:

$$
\begin{gathered}
L_{0} \rightarrow \chi_{0} \rightarrow A_{0} \\
L_{n-2} \rightarrow \chi_{n-2} \rightarrow \chi_{n-2} \rightarrow A_{1} \\
L_{n-1} \rightarrow \chi_{n-1} \rightarrow A_{n-1} .
\end{gathered}
$$

We define a sequence of operators $\left\{U_{n}\right\}_{n=0}^{\infty}$ as follows:

$$
U_{0}=1
$$

and, for $n>0$,

$$
U_{n} \stackrel{\text { def }}{=} A_{n-1} U_{n-1} \text {. }
$$

The operator $L_{n}$ is related to $L_{0}$ by means of $U_{n}$,

$$
L_{n} U_{n}=U_{n} L_{0} .
$$

So, $U_{n}$ intertwines the operators $L_{n}$ and $L_{0}$. We remark that this formalism follows very closely that of [1], which starts from $-\partial_{x}^{2}$ and generates the potentials in the manifold of rational solutions of $\mathrm{KdV}$.

The attentive reader might have noticed that the previous construction of $L_{n}$ is not complete if we do not have a mechanism for generating the elements in the kernel of $L_{n-1}$. Fortunately, such mechanism is given by the formula

$$
\chi_{n-1}=\frac{\alpha}{\chi_{n-2}}+\frac{\beta}{\chi_{n-2}} \int^{x} \chi_{n-2}^{2}(s) d s
$$

where $\chi_{n-2} \in \operatorname{ker} L_{n-2}$. The proof of this is elementary. Indeed, from $L_{n-1}=A_{n-2} A_{n-2}^{*}$ it follows that $1 / \chi_{n-2} \in \operatorname{ker} L_{n-2}$. Equation (48) is just the requirement that the Wronskian of $\chi_{n-1}$ and $1 / \chi_{n-2}$ be a constant.

Since

$$
v_{n-1}=\frac{\chi_{n-1}^{\prime}}{\chi_{n-1}}
$$

and

$$
u_{n}=v_{n-1}^{2}-v_{n-1}^{\prime},
$$

it follows that each step of the Darboux method introduces a new parameter, which is essentially the ratio $\beta / \alpha$ in Eq. (48). In the forthcoming construction, instead of using (48) we shall actually exhibit elements in the kernel of $L_{n-1}$. 


\subsection{A Few Facts}

Let $U_{n}^{*}$ be the formal adjoint of the operator $U_{n}$ defined in (46). Then, we have the following elementary fact

Lemma 2. For $n \geqq 0$,

$$
L_{0}^{n}=U_{n}^{*} U_{n}
$$

and

$$
L_{n}^{n}=U_{n} U_{n}^{*} .
$$

Proof. We first prove (52) by induction. Suppose that

$$
L_{n-1}^{n-1}=U_{n-1} U_{n-1}^{*}
$$

then

$$
\begin{aligned}
L_{n}^{n} & =\left(A_{n-1} A_{n-1}^{*}\right) \ldots\left(A_{n-1} A_{n-1}^{*}\right) \\
& =A_{n-1}\left(A_{n-1}^{*} A_{n-1}\right) \ldots\left(A_{n-1}^{*} A_{n-1}\right) A_{n-1} \\
& =A_{n-1} U_{n-1} U_{n-1}^{*} A_{n-1}^{*} \\
& =U_{n} U_{n}^{*} .
\end{aligned}
$$

To prove (51), Eq. (47) implies that $L_{n}^{n} U_{n}=U_{n} L_{0}^{n}$, and hence $U_{n} U_{n}^{*} U_{n}=U_{n} L_{n}^{n}$. But since $U_{n}$ is a monic differential operator and the cancellation law holds for such operators [17], it follows that $U_{n}^{*} U_{n}=L_{0}^{n}$. Q.E.D.

Lemma 2 implies that the process of applying $n$ Darboux transformations leads to a factorization of $L_{0}^{n}$. It also implies that $\operatorname{ker} U_{n} \subset \operatorname{ker} L_{0}^{n}$. From Eq. (47) it follows that $\operatorname{ker} U_{n}$ is invariant by $L_{0}$, and in fact is nilpotent of degree less than $n$ in this space. Our next step is to construct $\operatorname{ker} L_{0}^{n}$ by exhibiting sequences $\left\{\psi_{j}^{ \pm}\right\}_{j=0}^{l-1}$ of functions satisfying

$$
L_{0} \psi_{j}^{ \pm}=\psi_{j-1}^{ \pm}
$$

with

$$
\psi_{0}^{ \pm}=x^{ \pm l+1 / 2} \in \operatorname{ker} L_{0} .
$$

For $j \leqq l$, the trick lies in noticing that [32]

$$
L_{0} x^{2 j \pm l+1 / 2}=\left[(2 j \pm l)^{2}-l^{2}\right] x^{2(j-1) \pm l+1 / 2} .
$$

We define $\mu_{0}^{ \pm}=1$ and

$$
\mu_{j}^{ \pm}=\frac{1}{(2 j+l)^{2}-l^{2}} \mu_{j-1}^{ \pm},
$$

for $1 \leqq j \leqq l-1$. Thus, we have that

$$
\psi_{j}^{ \pm} \stackrel{\text { def }}{=} \mu_{j}^{ \pm} x^{2 j \pm l+1 / 2},
$$

satisfies Eq. (53) for $j \leqq l-1$. To extend this for $j \geqq l$ we have to look at the " + " and the "-" cases separately. In the "+" case, Eqs. (54) and (55) can be used to define $\psi_{j}^{+}$for every $j \geqq 1$. To extend the definition of $\psi_{j}^{-}$, for $j \geqq l$, first we define $\alpha_{i}$ and $\beta_{i}$ 
recursively by setting $\alpha_{0}=\mu_{l-1}^{-} /(-2 l), \beta_{0}=0$, and for $i \geqq 1$ :

$$
\begin{gathered}
\alpha_{i}=\frac{-1}{4 i(l+i)} \alpha_{i-1}, \\
\beta_{i}=\frac{-1}{4 i(l+i)} \beta_{i-1}-\frac{(l+2 i)}{2 i(l+i)} \alpha_{i} .
\end{gathered}
$$

Second, we take, for $i \geqq 0$,

$$
\psi_{l+i}^{-} \stackrel{\text { def }}{=} \alpha_{i} x^{l+2 i+1 / 2} \log x+\beta_{i} x^{l+2 i+1 / 2} .
$$

It is easy to check that

$$
L_{0} \psi_{l+i}^{-}=\psi_{l+i-1}^{-} .
$$

Using Eqs. (53) and (56), it is straightforward to show that $\left\{\psi_{l}^{ \pm}\right\}_{l=0}^{n-1}$ is a basis for $\operatorname{ker} L_{0}^{n}$.

\subsection{Dominant and Recessive Solutions}

We shall next recall the concept of dominant and recessive solutions, which was crucial in [8] to construct the bispectral potentials of rank two. It was shown therein that if we apply $n \leqq l$ Darboux transformations to $L_{0}$, the resulting potential is such that the differential equation

$$
-\partial_{x}^{2} \varphi+u_{n} \varphi=0,
$$

has a regular singular point at $x=0$. Furthermore,

$$
u_{n} \sim \frac{\varrho^{2}-1 / 4}{x^{2}}
$$

as $x \rightarrow 0$, for some $\varrho \in \mathbf{Z}_{\geq 0}$. In this situation, the two roots of the indicial equation differ by an integer, and the Frobenius series representing the solutions of (57) might require logarithmic terms. In any case, (57) always admits one solution of the form $\varphi_{-}=x^{\varrho+1 / 2}(1+\mathcal{O}(x))$, as $x \rightarrow 0$, which is uniquely determined by this asymptotic behavior. Any nonzero multiple of this solution is called recessive. On the other hand, any other linearly independent solution is called dominant. In this case, however, the solution is not uniquely determined by its leading behavior, since adding $c \varphi$ _ to a dominant solution does not alter this leading behavior.

The manifold $M_{n}$ will be constructed by applying $n$ successive Darboux transformations to $L_{0}$, using at each step a dominant eigenfunction for the eigenvalue zero. Before completing such a construction, we still need a few general facts about differential operators.

The intertwining operator $U_{n}$ of Eq. (46) is monic and therefore can be written as [17]

$$
U_{n} \varphi=\frac{W\left[\varphi_{1}, \ldots, \varphi_{n}, \varphi\right]}{W\left[\varphi_{1}, \ldots, \varphi_{n}\right]},
$$

where $\operatorname{span}\left\{\varphi_{1}, \ldots, \varphi_{n}\right\}=\operatorname{ker} U_{n}$. If we compare both sides of the equation $L_{n} U_{n}=U_{n} L_{0}$, we obtain that

$$
u_{n}=u_{0}-2 \partial_{x}^{2} \log W_{n},
$$


where $W_{n}=W\left[\varphi_{1}, \ldots, \varphi_{n}\right]$. The facts that $\varphi_{i} \in \operatorname{ker} U_{n} \subset \operatorname{ker} L_{0}^{n}$, as we showed in Lemma 2, and that

$$
\operatorname{ker} L_{0}^{n}=\operatorname{span}\left\{\psi_{0}^{ \pm}, \ldots, \psi_{n-1}^{ \pm}\right\}
$$

imply that $W_{n}$ is a polynomial in $x^{1 / 2}$ and in $\log x$. Hence, the asymptotic behavior of $u_{n}$ in a sector near $x=\infty$ is

$$
u_{n}=\mathcal{O}\left(1 / x^{2}\right),
$$

and $u_{n} \in \mathscr{U}$. This remark is important because we want to be able to apply to $u_{n}$ nonlocal operators involving $\int_{\infty}^{x}$.

We are ready now to define the manifold $M_{n}$ by induction. It will be parametrized by ${ }^{5}$ the $n$-tuple $\left(t_{0}, \ldots, t_{n-1}\right) \in \mathbf{C}^{n}$. We start with

$$
\chi_{0} \stackrel{\text { def }}{=} U_{0}\left(\psi_{0}^{-}+t_{0} \psi_{0}^{+}\right)=\psi_{0}^{-}+t_{0} \psi_{0}^{+},
$$

which is obviously in $\operatorname{ker} L_{0}$. The operators $A_{0}, U_{1}$, and $L_{1}$ are defined as in Eqs. (43), (45), and (46), with $n=1$.

Suppose that $A_{j}, \chi_{j}, U_{j}$, and $L_{j}$ have been defined as in Eqs. (37), (38), (39), (41), and (42) with $\chi_{j}=U_{j}\left(\psi_{j}^{-}+t_{0} \psi_{j}^{+}+\ldots+t_{j} \psi_{0}^{+}\right) \in \operatorname{ker} L_{j}$ for $0 \leqq j \leqq n-2$. We take

$$
U_{n-1}=A_{n-2} U_{n-2}=\left(\partial_{x}-\frac{\chi_{n-2}^{\prime}}{\chi_{n-2}}\right) U_{n-2}
$$

and

$$
\chi_{n-1} \stackrel{\text { def }}{=} U_{n-1}\left(\psi_{n-1}^{-}+t_{0} \psi_{n-1}^{+}+\ldots+t_{n-1} \psi_{0}^{+}\right) .
$$

Now, we claim that $\chi_{n-1} \in L_{n-1}$. Indeed, first we note that

$$
L_{n-1} U_{n-1} \psi_{0}^{+}=U_{n-1} L_{0} \psi_{0}^{+}=0 \text {. }
$$

Second, we remark that

$$
\begin{aligned}
& L_{n-1} U_{n-1}\left(\psi_{n-1}^{-}+t_{0} \psi_{n-1}^{+}+\ldots+t_{n-2} \psi_{1}^{+}\right) \\
& \quad=A_{n-2} A_{n-2}^{*} A_{n-2} \ldots A_{0}\left(\psi_{n-1}^{-}+t_{0} \psi_{n-1}^{+}+\ldots+t_{n-2} \psi_{1}^{+}\right) \\
& \quad=A_{n-2} L_{n-2} U_{n-2}\left(\psi_{n-1}^{-}+t_{0} \psi_{n-1}^{+}+\ldots+t_{n-2} \psi_{1}^{+}\right) \\
& \quad=A_{n-2} U_{n-2} L_{0}\left(\psi_{n-1}^{-}+t_{0} \psi_{n-1}^{+}+\ldots+t_{n-2} \psi_{1}^{+}\right) .
\end{aligned}
$$

But since $L_{0} \psi_{j+1}^{ \pm}=\psi_{j}^{ \pm}$, we have that the right-hand side of the previous equation is

$$
A_{n-2} U_{n-2}\left(\psi_{n-2}^{-}+t_{0} \psi_{n-2}^{+}+\ldots+t_{n-2} \psi_{0}^{+}\right)=A_{n-2} \chi_{n-2}=0
$$

Putting Eqs. (62) and (63) together it follows that

$$
L_{n-1} \chi_{n-1}=0 \text {. }
$$

The next goal is to show that if $0 \leqq n \leqq l$, then at $x=0, U_{n-1}\left(\psi_{0}^{+}\right)$is recessive and $U_{n-1}\left(\psi_{n-1}^{-}+t_{0} \psi_{n-1}^{+}+\ldots+t_{n-2} \psi_{1}^{+}\right)$is dominant. The proof of this will be based on two lemmas.

${ }_{5}$ The use of the variables $t_{j}, j \geqq 0$, is due to Wright [32] 
Lemma 3. If $\chi$ and $f$ are holomorphic in an open sector $S$ near $x=0$, with asymptotics

$$
\chi=x^{y}(1+\mathcal{O}(x))
$$

and

$$
f=x^{\beta}(1+\mathcal{O}(x)),
$$

then, for $x$ in a subsector of $S$,

$$
\chi \partial_{x} \chi^{-1} f=(\beta-\gamma) x^{\beta-1}+\mathcal{O}\left(x^{\beta}\right) .
$$

The proof of this lemma is a straightforward computation. We also note that if $\beta \neq \gamma$, then Eq. (67) can be rewritten as

$$
\chi \partial_{x} \chi^{-1} f=(\beta-\gamma) x^{\beta-1}(1+\mathcal{O}(x)) .
$$

We are now ready to show:

Lemm 4. Let

$$
\varphi_{j}^{+} \stackrel{\text { def }}{=} U_{j}\left(\psi_{0}^{+}\right)
$$

and

$$
\varphi_{j}^{-} \stackrel{\text { def }}{=} U_{j}\left(\psi_{j}^{-}+t_{0} \psi_{j}^{+}+\ldots+t_{j-1} \psi_{1}^{+}\right) \text {. }
$$

If $0 \leqq j \leqq l-1$, then $\varphi_{j}^{+}$is recessive and $\varphi_{j}^{-}$is dominant at $x=0$.

Proof. We shall first show by induction that

$$
\chi_{j}=\lambda_{j} x^{\gamma_{j}}(1+\mathcal{O}(x))
$$

for $0 \leqq j \leqq l-1$, where $\gamma_{j}=j-l+1 / 2$ and $\lambda_{j} \neq 0$. Indeed, for $j=0$ this property is obviously satisfied. Suppose it is true up to $j-1$, let's prove it for $j$. But if we recall that

$$
\chi_{j}=U_{j}\left(\psi_{j}^{-}+t_{0} \psi_{j}^{+}+\ldots+t_{j} \psi_{0}^{+}\right),
$$

we get

$$
\chi_{j}=A_{j-1} \ldots A_{0}\left(\mu_{j}^{-} x^{2 j-l+1 / 2}(1+\mathcal{O}(x))\right)
$$

Using Lemma 4,

$$
\begin{aligned}
\chi_{j} & =\chi_{j-1} \partial_{x} \frac{1}{\chi_{j-1}} \chi_{j-2} \partial_{x} \frac{1}{\chi_{j-2}} \ldots \chi_{0} \partial_{x} \frac{1}{\chi_{0}}\left(\mu_{j}^{-} x^{2 j-l+1 / 2}(1+\mathcal{O}(x))\right) \\
& =\mu_{j}^{-} \lambda_{j} x^{j-l+1 / 2}(1+\mathcal{O}(x))
\end{aligned}
$$

where

$$
\lambda_{j}=\prod_{i=1}^{j}(2 j-2 i+2) \neq 0 .
$$

Therefore, $\chi_{j}=\lambda_{j} x^{\gamma_{j}}(1+\mathcal{O}(x))$, with $\gamma_{j}$ and $\lambda_{j}$ as above. A similar computation with $\varphi_{j}^{+}=U_{j}\left(\psi_{0}^{+}\right)$gives

$$
\varphi_{j}^{+}=2^{j} \prod_{i=1}^{j}(l-i+1) x^{l-j+1 / 2}(1+\mathcal{O}(x)) .
$$

Hence, for $0 \leqq j \leqq l-1, U_{j}\left(\psi_{0}^{+}\right)$is recessive and $\varphi_{j}^{-}=\chi_{j}-t_{j} \varphi_{j}^{+}$is dominant at $x=0$. Q.E.D. 
The consequence of the previous lemma is that for $0 \leqq j \leqq l$ the potential $u_{j}$ was obtained from $u_{0}=\left(l^{2}-1 / 4\right) / x^{2}$ using at the $j^{\text {th }}$ Darboux transformation a dominant element in the kernel of $L_{j-1}$.

If we try to apply more than $l$ Darboux transformations to $u_{0}=\left(l^{2}-1 / 4\right) / x^{2}$ we have to introduce logarithmic terms in the functions $\psi_{j}^{ \pm}$. The corresponding potentials cease to be rational and the asymptotics of the eigenfunctions get a little bit more complicated. However, the construction described above can be continued for arbitrary values of $n$. The only point that needs to be verified is that $\varphi_{j}^{+}=U_{j}\left(\psi_{0}^{+}\right)$and $\varphi_{j}^{-}=U_{j}\left(\psi_{j}^{-}+t_{0} \psi_{j}^{+}+\ldots+t_{j-1} \psi_{1}^{+}\right)$are linearly independent. This guarantees that at this step we are obtaining a generic element in the kernel of $L_{j-1}$. The following lemma provides a general proof of this fact and an alternative one for $j<l$.

Lemma 5. For every $j \geqq 0, \varphi_{j}^{+}$and $\varphi_{j}^{-}$, as defined in Eqs. (69) and (70), are linearly independent.

Proof. If $j=0$, this is immediate since $U_{0}=1$ and $\varphi_{0}^{ \pm}=\psi_{0}^{ \pm}$. Let's assume that $j \geqq 1$. If $\alpha \varphi_{j}^{-}+\beta \varphi_{j}^{+}=0$, then

$$
\alpha\left(\psi_{j}^{-}+t_{0} \psi_{j}^{+}+\ldots+t_{j-1} \psi_{1}^{+}\right)+\beta \psi_{0}^{+} \in \operatorname{ker} U_{j} .
$$

From Eq. (58) we have that $\operatorname{ker} U_{j}=\operatorname{span}\left\{\phi_{0}, \ldots, \phi_{j-1}\right\}$, with $\phi_{i}=\psi_{i}^{-}+t_{0} \psi_{i}^{+}+\ldots$ $+t_{i} \psi_{0}^{+}$. But $L_{0} \phi_{0}=0$ and $L_{0} \phi_{i}=\phi_{i-1}$ for $i \geqq 1$. So, if we write

$$
\alpha\left(\psi_{j}^{-}+t_{0} \psi_{j}^{+}+\ldots+t_{j} \psi_{1}^{+}\right)+\beta \psi_{0}^{+}=\sum_{i=0}^{j-1} \varepsilon_{i} \phi_{i},
$$

where $\varepsilon_{i} \in \mathbf{C}$, and apply $L_{0}^{j}$ to both sides we get $\alpha \psi_{0}=0$. Hence, $\alpha=0$. Since $\psi_{0}^{+} \notin \operatorname{ker} U_{j}$ for $j>0$, it follows that $\varphi_{j}^{+} \neq 0$, which implies that $\beta=0$. Q.E.D.

The statements 1 and 2 of the next proposition summarize the results so far. The results 3 and 4 can be found in $[8,32]$.

Proposition 6. The potential $u_{n}$ obtained by the preceding construction has the following properties:

1. We can write for any $n$

$$
u_{n}=\frac{l^{2}-1 / 4}{x^{2}}-2 \partial_{x}^{2} \log W_{n}
$$

with

$$
W_{n}=W\left[\psi_{0}^{-}+t_{0} \psi_{0}^{+}, \ldots, \psi_{n-1}^{-}+t_{0} \psi_{n-1}^{+}+\ldots+t_{n-1} \psi_{0}^{+}\right] .
$$

2. The asymptotic behavior of $u_{n}$ at $x=\infty$, in a sector of angle smaller than $2 \pi$ near to infinity, is $u_{n}=\mathcal{O}\left(1 / x^{2}\right)$.

3. (Duistermaat and Grünbaum [8]) For $0<n \leqq l$, the manifold

$$
M_{n} \stackrel{\text { def }}{=}\left\{u_{n}\left(\cdot ; t_{0}, \ldots, t_{n-1}\right) \mid\left(t_{0}, \ldots, t_{n-1}\right) \in C^{n}\right\}
$$

is composed of rank 2 bispectral potentials.

4. (Wright [32]) For $0<n \leqq l$, the most general potential obtained by a sequence of $n$ Darboux transformations using at the $j^{\text {th }}$ step, $0<j \leqq n$, a dominant element in $\operatorname{ker} L_{j-1}$ belongs to $M_{n}$. 


\subsection{The $m K d V$ Fields}

We shall now collect a few facts about the function $v_{n}$, which is given by

$$
v_{n}=\partial_{x} \log \chi_{n} .
$$

Since $\chi_{n}$ satisfies $L_{n} \chi_{n}=0$, it follows that

$$
u_{n}=v_{n}^{2}+v_{n}^{\prime} \text {. }
$$

It is also easy to check that

$$
u_{n+1}=u_{n}-2 \partial_{x}^{2} \log \chi_{n} .
$$

The function $v_{n}$ can be written explicitly as

$$
\begin{aligned}
v_{n} & =\partial_{x} \log \chi_{n} \\
& =\partial_{x} \log \frac{W\left[\phi_{n-1}, \ldots, \phi_{0}, \phi_{n}\right]}{W\left[\phi_{n-1}, \ldots, \phi_{0}\right]} \\
& =\partial_{x} \log \frac{W_{n+1}}{W_{n}} .
\end{aligned}
$$

We note that the field $v_{n}$ depends on $n+1$ variables $t_{0}, \ldots, t_{n}$, and not $n$ as $u_{n}$. In the next section the following lemma will play an important role:

Lemma 7. For every $n \geqq 1$, there exists $c_{n} \neq 0$, independent of $x$, such that

$$
\frac{\partial}{\partial t_{n}} \chi_{n}=\frac{c_{n}}{\chi_{n-1}} .
$$

Proof. From the definition of $\chi_{n}$ we have that

$$
\frac{\partial}{\partial t_{n}} \chi_{n}=U_{n}\left(\psi_{0}^{+}\right)
$$

We claim that $U_{n}\left(\psi_{0}^{+}\right) \in \operatorname{ker} A_{n-1}^{*}$. Indeed,

$$
\begin{aligned}
A_{n-1}^{*} U_{n}\left(\psi_{0}^{+}\right) & =A_{n-1}^{*} A_{n-1} U_{n-1} \psi_{0}^{+} \\
& =L_{n-1} U_{n-1} \psi_{0}^{+} \\
& =U_{n-1} L_{0} \psi_{0}^{+} \\
& =0 .
\end{aligned}
$$

But, $\operatorname{ker} A_{n-1}^{*}=\operatorname{span}\left\{1 / \chi_{n-1}\right\}$. For $n \leqq l$, since the function $U_{n}\left(\psi_{0}^{+}\right)$has nonzero asymptotics as $x \rightarrow 0$, it follows that $\exists c_{n} \in \mathbf{C} \backslash\{0\}$ such that (76) holds. This constant $c_{n}$ can be computed explicitly using the techniques of Lemmas 3 and 4. For $n>l$, the fact that $c_{n} \neq 0$ follows from the fact that $\psi_{0}^{+} \notin \operatorname{ker} U_{j}$. Q.E.D.

\section{The Tangency Property}

In this section we shall show that the vector fields $\tau_{j}, j \geqq 0$, are tangent to the manifold $M_{n}$ of potentials obtained by $n$ successive applications of Darboux to $u_{0}=\left(l^{2}-1 / 4\right) / x^{2}$. Our proof follows closely an ingenious argument of Adler and 
Moser [1], which was used to prove the tangency of the higher order $\mathrm{KdV}$ vector fields to the manifolds of rational solutions of $\mathrm{KdV}$. The key element in this proof is the relation among the $\mathrm{KdV}$ flows, the mKdV flows, the Miura map and the Darboux transformation.

Theorem 8. The vector fields $\tau_{j}$, defined by

$$
\tau_{0}(u)=\frac{1}{2} x u_{x}+u
$$

and

$$
\tau_{j}(u)=\mathbf{N}_{u}^{j} \tau_{0}
$$

are tangent to the manifold $M_{n}$.

Proof. We shall show by induction on $n$ that $\tau_{j}\left(u_{n}\right)$ is a linear combination of $\partial u_{n} / \partial t_{k}$, with $0 \leqq k \leqq n-1$. For $n=0$ this is trivial, since $u_{0}=\left(l^{2}-1 / 4\right) / x^{2}$, and $\tau_{0}\left(u_{0}\right)=0$, which implies that $\tau_{j}\left(u_{0}\right)=0$, for $j \geqq 0$. Suppose that we have established the existence of coefficients $\gamma_{j k}\left(t_{0}, \ldots, t_{n-1}\right)$, independent of $x$ (but possibly depending on $n$ ) such that

$$
\tau_{j}\left(u_{n}\right)=\sum_{k=0}^{n-1} \gamma_{j k} \frac{\partial}{\partial t_{k}} u_{n} .
$$

Since $u_{n}$ is related to $v_{n}$ via the Miura map $F(v)=v^{2}+v_{x}$, we have that

$$
\left(2 v_{n}+\partial_{x}\right) \sigma_{j}\left(v_{n}\right)=\tau_{j}\left(u_{n}\right)=\sum_{k=0}^{n-1} \gamma_{j k} \frac{\partial}{\partial t_{k}} u_{n} .
$$

Here, $\sigma_{j}$ is the $j^{\text {th }}$ master symmetry field associated to the $\mathrm{mKdV}$ hierarchy as defined in Sect. 2. But,

$$
\frac{\partial}{\partial t_{k}} u_{n}=\left(2 v_{n}+\partial_{x}\right) \frac{\partial}{\partial t_{k}} v_{n}
$$

and hence

$$
\left(2 v_{n}+\partial_{x}\right) \sigma_{j}\left(v_{n}\right)=\left(2 v_{n}+\partial_{x}\right) \sum_{k=0}^{n-1} \gamma_{j k} \frac{\partial}{\partial t_{k}} v_{n}
$$

Therefore,

$$
\sigma_{j}\left(v_{n}\right)-\sum_{k=0}^{n-1} \gamma_{j k} \frac{\partial}{\partial t_{k}} v_{n} \in \operatorname{ker}\left(\partial_{x}+2 v_{n}\right) .
$$

Now, $\operatorname{ker}\left(\partial_{x}+2 v_{n}\right)$ is 1-dimensional and generated by $1 / \chi_{n}^{2}$ because of Eq. (73). Therefore, we can write

$$
\sigma_{j}\left(v_{n}\right)-\sum_{k=0}^{n-1} \gamma_{j k} \frac{\partial}{\partial t_{k}} v_{n}=\frac{\beta}{\chi_{n}^{2}},
$$

where the constant $\beta$ is independent of $x$. Our next task is to rewrite the right-hand side of (77) as $\gamma_{j n} \partial v_{n} / \partial t_{n}$. In order to do that we compute

$$
\frac{\partial}{\partial t_{n}} v_{n}=\frac{\partial}{\partial t_{n}} \frac{\partial}{\partial x} \log \chi_{n}=\frac{\partial}{\partial x}\left(\frac{1}{\chi_{n}} \frac{\partial}{\partial t_{n}} \chi_{n}\right) .
$$


Using Lemma 7 we get that

$$
\frac{\partial}{\partial t_{n}} v_{n}=c_{n} \frac{\partial}{\partial x}\left(\frac{1}{\chi_{n}} \frac{1}{\chi_{n-1}}\right)
$$

for some nonzero $c_{n}$. Hence,

$$
\frac{\partial}{\partial t_{n}} v_{n}=c_{n} \frac{1}{\chi_{n}^{2}} W\left[\chi_{n}, \chi_{n-1}^{-1}\right]
$$

We recall from the previous section that $\chi_{n}$ and $\chi_{n-1}^{-1}$ are linearly independent solutions of $L_{n} \varphi=0$. Therefore, the Wronskian $W\left[\chi_{n}, \chi_{n-1}^{-1}\right]$ is a nonzero constant independent of $x$. So we can write

$$
\frac{\beta}{\chi_{n}^{2}}=\gamma_{j n} \frac{\partial}{\partial t_{n}} v_{n},
$$

where $\gamma_{j n}$ is also independent of $x$. Equations (77) and (78) give that

$$
\sigma_{j}\left(v_{n}\right)=\sum_{k=0}^{n} \gamma_{j k} \frac{\partial}{\partial t_{k}} v_{n} .
$$

We conclude using that $u_{n+1}=v_{n}^{2}-v_{n}^{\prime}$ and that $\mathbf{M}_{v}$ is invariant by reflections $v \mapsto-v$. Therefore,

$$
\begin{aligned}
\tau_{j}\left(u_{n+1}\right) & =\tau_{j}\left(v_{n}^{2}-v_{n}^{\prime}\right) \\
& =\left(\partial_{x}-2 v_{n}\right) \sigma_{j}\left(-v_{n}\right) \\
& =-\left(\partial_{x}-2 v_{n}\right) \sigma_{j}\left(v_{n}\right) \\
& =\left(2 v_{n}-\partial_{x}\right) \sum_{k=0}^{n} \gamma_{j k} \frac{\partial}{\partial t_{k}} v_{n} \\
& =\sum_{k=0}^{n} \gamma_{j k} \frac{\partial}{\partial t_{k}} u_{n+1} \text { Q.E.D. }
\end{aligned}
$$

Remarks. 1. The Darboux transformation that sends $u_{n} \mapsto \tilde{u}=u_{n+1}$, changes the sequence $\gamma_{j k}$ as follows:

$$
\tilde{\gamma}_{j k}= \begin{cases}\gamma_{j k} & \text { for } 0 \leqq k \leqq n-1, \\ \text { function of }\left(t_{0} \ldots t_{n}\right) & \text { for } k=n, \\ 0 & \text { for } k>n .\end{cases}
$$

2. We can construct a family of rational solutions of the master symmetry fields by solving equations of the form:

$$
\frac{d t_{k}}{d s}=\gamma_{j k}\left(t_{0}, \ldots, t_{n}\right)
$$

for $0 \leqq k \leqq n$. However, since the master symmetry flows do not commute with one another, we cannot expect to be able to find new time variables $s_{0}, \ldots, s_{n}$ such that

$$
\frac{\partial t_{k}}{\partial s_{k}}=\gamma_{j k}\left(t_{0}, \ldots, t_{n}\right),
$$

as in the case of the KdV hierarchy [1]. Indeed, we can check that such variables do not exist in a few examples. 
3. It is not hard to adapt the reasoning in the proof of Theorem 8 to the case of $u_{0}$ of the form $v(v-1) / x^{2}$ for $v$ a natural number. The manifolds generated by successive applications of Darboux to such potentials are composed of AdlerMoser potentials. Therefore, the master symmetries are also tangent to the manifolds of rational solutions of KdV that decay at infinity. Now, the manifolds of Adler-Moser potentials are contained in the class of bispectral potentials [8]. When the latter manifolds are united with the manifolds $M_{n, l}$, for $0 \leqq n<l$, they exhaust, modulo translation, the class of bispectral potentials that decay at infinity [8]. Therefore, the master symmetries of $\mathrm{KdV}$ are tangent to all the manifolds that satisfy the class of bispectral potentials decaying at infinity.

The results obtained above can be translated in the "mKdV domain." We define the manifold $M_{n}^{\prime}$ by

$$
M_{n}^{\prime}=\left\{v_{n}\left(\cdot ; t_{0}, \ldots, t_{n}\right) \mid\left(t_{0}, \ldots, t_{n}\right) \in \mathbf{C}^{n+1}\right\} .
$$

One simple consequence of the proof of Theorem 8 is:

Corollary 9. The vector fields $\sigma_{j}$, defined by

$$
\sigma_{0}(u)=\frac{1}{2}(x u)_{x}
$$

and

$$
\sigma_{j}(u)=\mathbf{M}_{v}^{j} \sigma_{0},
$$

are tangent to the manifold $M_{n}^{\prime}$.

We conclude this section with a small digression on the matrix case of the bispectral problem. In $[33,34]$ the bispectral problem was introduced for matrix differential operators. The question is analogous to the one formulated in Sect. 1, except that one now allows the operator $B\left(\lambda, \partial_{\lambda}\right)$ to have matrix coefficients, and requires its leading order coefficient to be nonsingular. The remark is that the matrix differential operator

$$
\mathbf{L}=\left[\begin{array}{cc}
0 & \partial_{x}+v_{n} \\
\partial_{x}-v_{n} & 0
\end{array}\right]
$$

possesses the bispectral property. The case $n=1$ was proved in [34] as a consequence of a more general result for matrix differential operators. The case of arbitrary $n$ can be obtained as a consequence of the results in [8]. Details will appear elsewhere.

\section{Final Remarks}

The main motivation for the present work was to understand the results of [8] in terms of hierarchies of nonlinear evolution equations. This can also be looked upon as part of a long term program of attacking the bispectral problem in the case where $L$ is a matrix differential operator or a scalar differential operator of order greater than two. Some initial steps have been done in this direction in related works $[14,33-35]$. We believe that similar results to the ones described here concerning the hierarchy of master symmetries of KdV should also hold in the higher order case. We are currently pursuing some of these avenues. 
We close with some general remarks:

It is interesting to note that if one looks at the bispectral problem as stated in Sect. 1, one very trivial symmetry appears: If $u(x)$ is bispectral, then so is $u\left(x+x_{0}\right)$. In other words, $X_{0}(u)=u_{x}$ is the infinitesimal generator of a one-parameter group of symmetries for the class of bispectral potentials. Another symmetry, which is not so obvious is the following: If $u(x)$ is bispectral, then so is the potential $t^{2} u(x t)$ for any value of $t$. This transformation is induced by a change of scale in the Schrödinger operator, namely $x \mapsto t x$. It turns out that the infinitesimal generator of the one-parameter group of symmetries

$$
S(t): u(x) \mapsto t^{2} u(x t)
$$

is exactly $2 \tau_{0}(u)=x u_{x}+2 u$. To check this just differentiate $S(t) u$ at $t=1$. The remarkable fact, which certainly deserves further investigation, is that the hierarchies of vector fields obtained by applying the Nijenhuis tensor $\mathbf{N}_{u}$ to $\tau_{0}$ and to $X_{0}$ are deeply connected to the bispectral problem. As we showed in this paper the elements in the hierarchy $\left\{\tau_{j}\right\}_{j \geqq 0}$ are tangent to the manifolds of rank two bispectral potentials. This, added to the earlier result of Duistermaat and Grünbaum [8], which says that the manifolds of rational solutions of the $\mathrm{KdV}$ hierarchy $\left\{X_{j}\right\}_{j \geq 0}$ are also bispectral, indicates that there should be some more general theory lurking in the background of the results in [8,14, 33-35].

Another remark of interest is that the tangency result of Sect. 4 also holds for the manifolds of rational solutions of KdV. In other words, the manifolds of rational solutions of $\mathrm{KdV}$ are also invariant by the flows of the master symmetry hierarchy $\left\{\tau_{j}\right\}_{j \geq 0}$. The proof of this follows the same lines of the proof in Sect. 4 . This yields the following picture:

Rank 2 Case

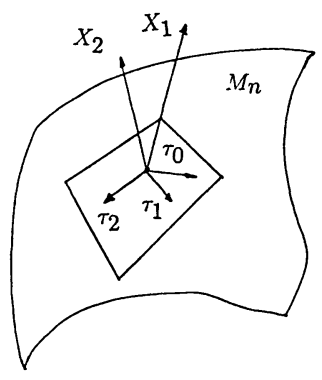

Rank 1 Case

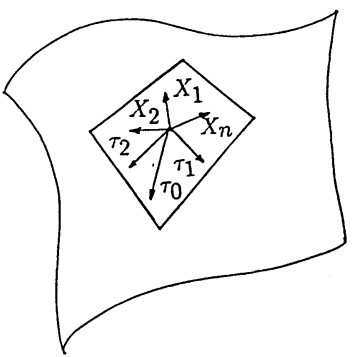

Fig. 1. The vector fields in the master symmetry hierarchy are tangent to both the rank 1 and rank 2 bispectral potentials. The vector fields in the $\mathrm{KdV}$ hierarchy are tangent only to rank 1 bispectral potentials

In [15] a differential equation was found, which is very similar to $u_{t}=\tau_{1}(u)$. It works for the potentials obtained by applying Darboux once to $u_{0}=3 / 4 x^{2}$. The coefficients of this equation, however, do not seem to be the appropriate ones for the constructions of the whole hierarchy by means of a recursion operator. 


\section{References}

1. Adler, M., Moser, J.: On a class of polynomials connected with the $\mathrm{KdV}$ equations. Commun. Math. Phys. 61, 1-30 (1978)

2. Airault, H., Mc Kean, H., Moser, J.: Rational and elliptic solutions of the KdV equation and a related many body problem. Commun. Pure Appl. Math. 30, 95-148 (1977)

3. Bishop, R., Goldberg, S.: Tensor analysis on manifolds. New York: Dover 1980

4. Chen, H., Lee, Y., Lin, J.: Preprint PL83-002, University of Maryland 1982

5. Crum, M.: Associated Sturm-Liouville systems. Quart. J. Math., Ser. 2 6, 121-127 (1955)

6. Darboux, G.: Leçons sur la Théorie Générale de Surface et les Applications Géométriques du Calcul Infinitésimal, Deuxième Partie. Paris, France: Gauthiers-Villars 1889

7. Deift, P., Trubowitz, E.: Inverse scattering on the line. CPAM 32, 121-251 (1977)

8. Duistermaat, J.J., Grünbaum, F.A.: Differential equations in the spectral parameter. Commun. Math. Phys. 103, 177-240 (1986)

9. Fröhlicher, A., Nijenhuis, A.: Theory of vector-valued differential forms, Part I. Indag. Math., t. 18, 338-350 (1956)

10. Fuchssteiner, B.: Mastersymmetries, higher order time-dependent symmetries and conserved densities of nonlinear evolution equations. Prog. Theor. Phys. 70 (6), 1508-1522 (1983)

11. Fuchssteiner, B.: Mastersymmetries for completely integrable systems in statistical mechanics. In: Garrido, L. (ed.), Proc. Sitges Conference. Lecture Notes in Physics, vol. 216, pp. 305-315. Berlin, Heidelberg, New York: Springer 1984

12. Gel'fand, I.M., Dikii, L.A.: Fractional powers of operators and Hamiltonian systems. Funkt. Anal. Pril. 10 (4), 13-29 (1976)

13. Gel'fand, I.M., Dikii, L.A.: Resolvents and Hamiltonian systems. Funkt. Anal. Pril. 11 (2), 11-27 (1977)

14. Grünbaum, F.A.: Differential equations in the spectral parameter: the higher order case. In: Proceedings of the conference on Tomographic Inverse Problems, Montpellier, pp. 307-322 (1986)

15. Grünbaum, F.A.: Some nonlinear evolution equations and related topics arising in medical imaging. Physica D 18, 308-311 (1986)

16. Helgason, S.: Differential geometry and symmetric spaces. New York: Academic Press 1962

17. Ince, E.L.: Ordinary differential equations. New York: Dover 1956

18. Lang, S.: Differential manifolds. Reading, Mass.: Addison-Wesley 1972

19. Lax, P.: Integrals of nonlinear equations of evolution and solitary waves. Commun. Pure Appl. Math. 31, 467-490 (1968)

20. Levi, D.: Hierarchies of nonlinear integrable evolution equations with variable coefficients. In: Leon, Jérôme J.P. (ed.), Workshop on Nonlinear Evolution Equations and Dynamical Systems, Montpellier, p. 75-85. Singapore: World Scientific 1986

21. Levi, D., Ranisco, O., Sym, A.: Dressing method vs. classical Darboux transformations. Il Nuovo Cim. 83 B(1), 34-42 (1984)

22. Magri, F.: Equivalence transformations for nonlinear evolution equations. J. Math. Phys. 18 (7), 1405-1411 (1977)

23. Magri, F.: A simple model of the integrable Hamiltonian equation. J. Math. Phys. 19, 1156-1162 (1978)

24. Magri, F., Kosmann-Schwarzbach, Y.: Poisson-Nijenhuis structures. To appear

25. Matveev, V.B., Salle, M.A., Rybin, A.V.: Darboux transformations and coherent interaction of the light pulse with two level media. Inverse Problems 4, 173-183 (1988)

26. Oevel, G., Fuchssteiner, B., Blaszak, M.: Action-Angle representation of multisolitons by potentials of mastersymmetries. Prog. Theor. Phys. 83 (3), 395-413 (1990)

27. Oevel, W., Falck, M.: Master symmetries for finite dimensional integrable systems. Prog. Theor. Phys. 75 (6), 1328-1341 (1986)

28. Oevel, W., Fuchssteiner, B.: Explicit formulas for symmetries and conservation laws of the Kadomtsev-Petviashvili equation. Phys. Lett. 88 A(7), 323-327 (1982)

29. Olver, P.: Applications of Lie groups to differential equations. Berlin, Heidelberg, New York: Springer 1986

30. Segal, G., Wilson, G.: Loop groups and equations of KdV type. Publ. Math. IHES 61, 5-65 (1985) 
31. Wasow, W.: Asymptotic expansions for ordinary differential equations. New York: Dover 1987

32. Wright, P.E.: Darboux transformations, algebraic varieties of Grassmann manifolds, commuting flows and bispectrality. PhD thesis, University of California, Berkeley, 1987

33. Zubelli, J.P.: Differential equations in the spectral parameter for matrix differential operators of AKNS type. PhD thesis, University of California, Berkeley, 1989

34. Zubelli, J.P.: Differential equations in the spectral parameter for matrix differential operators. Physica D 43, 269-287 (1990)

35. Zubelli, J.P.: Rational solutions of nonlinear evolution equations, vertex operators and bispectrality. J. Differ. Eqs. (to appear)

Communicated by A. Jaffe 
